\title{
Pseudomonas infection and mucociliary and absorptive clearance in the cystic fibrosis lung
}

\author{
Landon W. Locke1, Michael M. Myerburg ${ }^{1}$, Daniel J. Weiner², \\ Matthew R. Markovetz ${ }^{3}$, Robert S. Parker ${ }^{3,4,5,6}$, Ashok Muthukrishnan?, \\ Lawrence Weber ${ }^{7}$, Michael R. Czachowski ${ }^{8}$, Ryan T. Lacy ${ }^{4}$, \\ Joseph M. Pilewski ${ }^{1,2,9}$ and Timothy E. Corcoran ${ }^{1,3,4}$
}

\begin{abstract}
Affiliations: ${ }^{1}$ Pulmonary, Allergy, and Critical Care Medicine, University of Pittsburgh, Pittsburgh, PA, USA. ${ }^{2}$ Pulmonary Medicine, Allergy, and Immunology, Children's Hospital of Pittsburgh, Pittsburgh, PA, USA. ${ }^{3}$ Dept of Chemical and Petroleum Engineering, University of Pittsburgh, Pittsburgh, PA, USA. ${ }^{4}$ Dept of Bioengineering, University of Pittsburgh, Pittsburgh, PA, USA. ${ }^{5}$ Dept of Critical Care Medicine, University of Pittsburgh, Pittsburgh, PA, USA. ${ }^{6}$ McGowan Institute for Regenerative Medicine, University of Pittsburgh Medical Center, Pittsburgh, PA, USA. ${ }^{7}$ Dept of Radiology, University of Pittsburgh Medical Center, Pittsburgh, PA, USA. ${ }^{8}$ Dept of Radiology, Children's Hospital of Pittsburgh, Pittsburgh, PA, USA. ${ }^{9}$ Dept of Cell Biology, University of Pittsburgh, Pittsburgh, PA, USA.
\end{abstract}

Correspondence: Tim Corcoran, University of Pittsburgh, UPMC MUH NW628, 3459 Fifth Ave, Pittsburgh, PA 15213, USA. E-mail: corcorantedupmc.edu

ABSTRACT Airway surface liquid hyperabsorption and mucus accumulation are key elements of cystic fibrosis lung disease that can be assessed in vivo using functional imaging methods. In this study we evaluated experimental factors affecting measurements of mucociliary clearance (MCC) and smallmolecule absorption (ABS) and patient factors associated with abnormal absorption and mucus clearance.

Our imaging technique utilises two radiopharmaceutical probes delivered by inhalation. Measurement repeatability was assessed in 10 adult cystic fibrosis subjects. Experimental factors were assessed in 29 adult and paediatric cystic fibrosis subjects (51 scans). Patient factors were assessed in a subgroup with optimal aerosol deposition (37 scans; 24 subjects). Paediatric subjects $(n=9)$ underwent initial and 2-year follow-up scans. Control subjects from a previously reported study are included for comparison.

High rates of central aerosol deposition influenced measurements of ABS and, to a lesser extent, MCC. Depressed MCC in cystic fibrosis was only detectable in subjects with previous Pseudomonas aeruginosa infection. Cystic fibrosis subjects without $P$. aeruginosa had similar MCC to control subjects. Cystic fibrosis subjects had consistently higher ABS rates.

We conclude that the primary experimental factor affecting MCC/ABS measurements is central deposition percentage. Depressed MCC in cystic fibrosis is associated with $P$. aeruginosa infection. ABS is consistently increased in cystic fibrosis.

@ERSpublications

Depressed mucus clearance in cystic fibrosis was only detectable in subjects with Pseudomonas aeruginosa infection http://ow.ly/YggZy

This article has supplementary material available from erj.ersjournals.com

Received: Nov 112015 | Accepted after revision: Feb 032016 | First published online: March 242016

This study is registered at clinicaltrials.gov with identifier numbers NCT01486199, NCT01887197, NCT00541190 and NCT01223183.

Support statement: This project was supported by the National Institutes of Health (NIH) (R01 HL108929 and P30 DK072506) and the Cystic Fibrosis Foundation Research Development Program to the University of Pittsburgh. The content is solely the responsibility of the authors and does not necessarily represent the official views of the National Heart, Lung, and Blood Institute or the NIH. Funding information for this article has been deposited with FundRef.

Conflict of interest: Disclosures can be found alongside the online version of this article at erj.ersjournals.com

Copyright OERS 2016 


\section{Introduction}

Cystic fibrosis is caused by mutations in the cystic fibrosis transmembrane conductance regulator (CFTR) gene, which encodes an epithelial anion channel [1]. In the cystic fibrosis airway, absent or dysfunctional CFTR limits chloride and bicarbonate secretion into the airway surface liquid (ASL). Hyperabsorption of sodium ions through the epithelial sodium channel also occurs, creating osmotic gradients that favour ASL and mucus dehydration [2-4]. The accumulation of dehydrated mucus is thought to lead to patterns of chronic infection, inflammation, bronchiectasis and premature respiratory failure. Measurements of mucociliary clearance (MCC) using nuclear imaging techniques are used in cystic fibrosis therapeutics development [5-9]. Our centre has developed a variation of this technique that includes a parallel measurement of small molecule absorption (ABS) [10-13]. In vitro studies have demonstrated a proportional relationship between ABS and ASL absorption rate [11]. ABS is increased in the cystic fibrosis lung, and the inhalation of hypertonic saline has been shown to decrease ABS (ASL absorption) and increase MCC (mucus clearance) [12]. In this study we evaluated experimental factors affecting measurements of MCC and ABS and patient factors associated with abnormal absorption and mucus clearance in adult and paediatric subjects with cystic fibrosis.

\section{Materials and methods}

\section{Experimental factors affecting measurements of MCC/ABS}

We assessed measurement repeatability in a group of 10 adult cystic fibrosis subjects who performed two MCC/ABS imaging measurements separated by 25-35 days. We determined the effects of aerosol deposition pattern using a combined analysis group including 51 scans from 29 cystic fibrosis subjects. This included measurements from: 1) the repeatability group (both measurements used); 2) a paediatric group of nine subjects who performed two MCC/ABS scans separated by a period of 22-26 months; and 3 ) a therapy group of 14 adult cystic fibrosis subjects who performed two MCC/ABS study days separated by 5-24 days (only baseline measurements were used). The first measurement from the paediatric study has been previously reported, along with outcomes from the therapy group [12]. Imaging procedures were identical during all studies. Approval was granted by the University of Pittsburgh institutional review board.

\section{Patient factors associated with abnormal MCC/ABS}

We assessed correlations between MCC/ABS and patient factors in the combined analysis group and in a subset of this group that had $\leqslant 50 \%$ deposition in central lung zones, the optimal deposition group, which included 37 scans from 24 cystic fibrosis subjects. In addition, comparisons were made with healthy control subjects from a previous study $(n=9)$. Patient factors included forced expiratory volume in $1 \mathrm{~s}$ (FEV1), forced vital capacity (FVC) and forced expiratory flow at $25-75 \%$ of FVC (FEF25-75\%), which were assessed immediately prior to each imaging study. For the paediatric cohort, the slopes of FEV1, FEF25-75\% and FVC \% predicted were also calculated by linear regression using available study and clinical pulmonary function tests recorded over the period from 2 years prior to the first imaging visit through the second imaging visit. The slopes are represented as a percentage change in predicted value per year. Sputum and deep oropharyngeal culture results, obtained for routine cystic fibrosis longitudinal care, were surveyed for all subjects. We also assessed whether subjects had previously demonstrated colonisation with Pseudomonas aeruginosa. Subjects were considered to be $P$. aeruginosa-positive if they had a single positive culture over the 2-year period before each imaging study.

\section{Radiopharmaceutical aerosol inhalation and imaging protocol}

Evaluation of ABS and MCC was performed as previously described [12]. Briefly, a liquid aerosol containing a nonabsorbable radiolabelled particle probe (technetium 99m sulfur colloid (Tc-SC), $296 \mathrm{MBq}$ ) and an absorbable radiolabeled small molecule (indium 111 DTPA (In-DTPA), $55 \mathrm{MBq}$ ) was inhaled by the subjects using a fixed breathing pattern to achieve predominant airways deposition [9]. (More detail is included in the online supplementary material.) Tc-SC is only cleared by MCC, while In-DTPA is cleared by both MCC and absorption. Subtracting the MCC clearance rate from the total DTPA clearance rate provides an estimate of the absorptive component of DTPA clearance (ABS) [10,12, 13]. Studies in epithelial cell cultures have demonstrated proportional relationships between ABS and ASL absorption. However, the transport of DTPA is mediated by paracellular liquid flows across the epithelium [11], and other factors such as changes in tight junction permeability or epithelial damage may also affect ABS. During the baseline measurement technique, subjects inhaled isotonic saline from $\mathrm{t}=10 \mathrm{~min}$ through $\mathrm{t}=20 \mathrm{~min}$, followed by 60 additional minutes of recumbent imaging.

\section{Statistical analysis}

Stata 12.1 (StataCorp, College Station, TX, USA) was used to perform all analysis. Nonparametric testing was used for group comparisons. For matched-pair data, the Wilcoxon matched-pairs signed-rank test was used (SIGNRANK in Stata). For unmatched data, Mann-Whitney testing was used (RANKSUM). 
Intraclass correlation (ICC) was used to compare measures in the repeatability portion of the study. A one-way random-effects model was used. Linear regression was used to compare potentially correlated continuous variables (REGRESS).

\section{Results}

\section{Experimental factors affecting measurements of MCC/ABS}

Characteristics of the 10 adult cystic fibrosis subjects who performed repeatability studies are summarised in table 1. Subjects performed two studies within a 25-30-day window, except for one subject whose studies were separated by 44 days due to illness. Measures of pulmonary function were similar between testing days (FEV1 \% pred $\mathrm{p}=0.30$; FVC $\%$ pred $\mathrm{p}=0.26$; and $\mathrm{FEF} 25-75 \% \%$ pred $\mathrm{p}=0.20$; using the Wilcoxon test), although three subjects did have a $>5 \%$ change in FEV1 \% pred. There were no statistical differences in indicators of deposition pattern between study days: central deposition percentage $(\mathrm{p}=0.24)$ and central/ peripheral deposition ratio $(\mathrm{p}=0.33)$.

The mean $\pm \mathrm{SD}$ ABS including all 20 measurements was $23.7 \pm 11.0 \% \cdot 80 \mathrm{~min}^{-1}$ and MCC was $32.7 \pm 15.8 \% \cdot 80 \mathrm{~min}^{-1}$. ABS and MCC values are compared in figure 1. Bland-Altman plots are shown in online supplementary figures S1 and S2. The mean \pm SD intrasubject difference between ABS measurements (day 2-day 1) was $5.4 \pm 8.4 \% \cdot 80 \mathrm{~min}^{-1}$ (ICC 0.63). Excluding data from the three subjects with a $>5 \%$ change in FEV1 between testing days reduced the mean difference to $3.4 \pm 5.2 \% \cdot 80 \mathrm{~min}^{-1}$ and increased ICC to 0.85 . The mean intrasubject difference between MCC measurements was $-1.5 \pm 12.0 \% \cdot 80 \mathrm{~min}^{-1}$ (ICC 0.75). Excluding data from the subjects with a $>5 \% \mathrm{FEV}_{1}$ change between visits changed the mean difference to $-4.2 \pm 7.7 \% \cdot 80 \mathrm{~min}^{-1}$ and increased ICC to 0.90 .

We analysed a combined dataset including data from three different studies in which 29 patients with cystic fibrosis (nine paediatric and 20 adult) performed one to three measurements each (a total of 51 measurements). As shown in figure 2, there was a positive linear correlation between central lung deposition and MCC and a negative linear correlation between central lung deposition and ABS. Very high central deposition favours rapid MCC and prevents accurate measurement of ABS, as the small molecule probe is cleared through MCC before it can be absorbed. The confounding effect of central deposition on ABS becomes less significant if 14 measurements with central deposition $>50 \%$ are removed ( $p$-value for linear regression increases to 0.10 ). The confounding effect on MCC becomes statistically insignificant if studies with central deposition $>55 \%$ are removed (four measurements). We generated an optimal deposition dataset by applying a conservative 50\% central deposition threshold to all 51 imaging measurements. This dataset included 37 measurements from 24 subjects. No healthy control subjects demonstrated central deposition $>50 \%$.

\section{Patient factors associated with abnormal MCC/ABS}

In figure 3, measurements of ABS and MCC from our combined (51 measurement) and optimal deposition (37 measurement) cystic fibrosis groups are compared to data from control subjects [12]. Our previous studies, which included these control subjects and a portion of the current cystic fibrosis subjects, concluded that ABS was significantly increased in cystic fibrosis and that MCC rates were similar in cystic fibrosis and

TABLE 1 Characteristics, pulmonary function, imaging outcomes and deposition variables from 10 adult cystic fibrosis subjects enrolled in the repeatability study group



Data are presented as $n$, unless otherwise stated. FEV1: forced expiratory volume in $1 \mathrm{~s}$; FEF25-75\%: forced expiratory flow at $25-75 \%$ of forced vital capacity; MCC: mucociliary clearance; ABS: DTPA absorption; M: male; F: female. " : change of $>5 \%$ between testing days. 

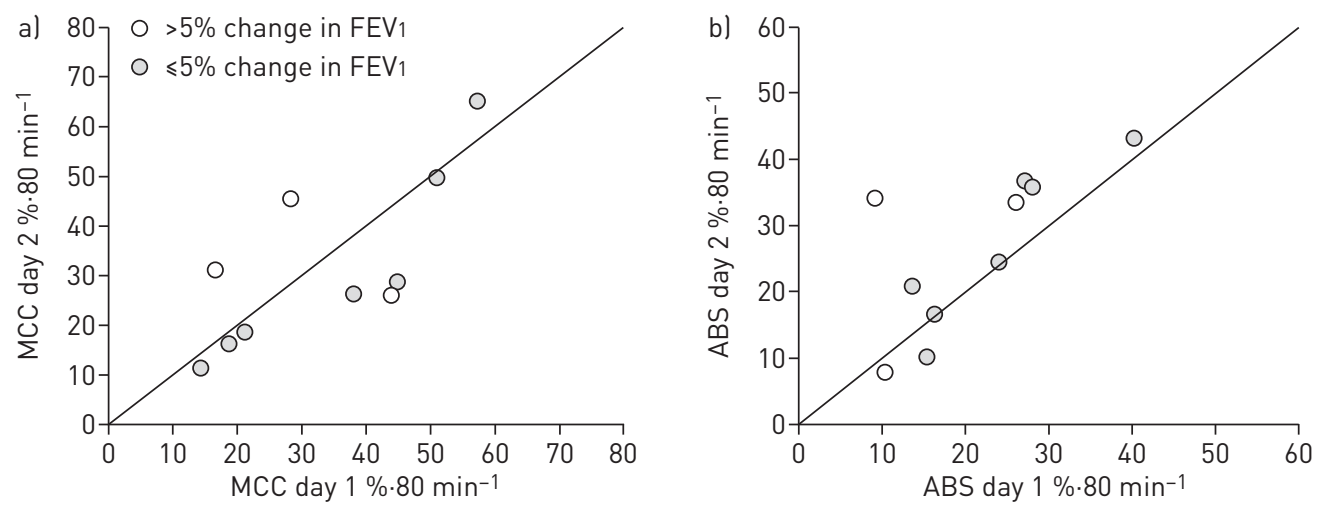

FIGURE 1 Measurement repeatability of a) mucociliary clearance (MCC) and b) DTPA absorption (ABS) in 10 cystic fibrosis subjects. FEV1: forced expiratory volume in $1 \mathrm{~s}$.

control subjects [12]. The present result corroborates that ABS is increased in cystic fibrosis, and with increased significance when the optimised deposition group is considered. MCC differences between cystic fibrosis and noncystic fibrosis approach significance only when the cystic fibrosis group with optimised deposition is considered $(\mathrm{p}=0.09$ ). The mean \pm SD FEV1 in the optimal deposition group was $82 \pm 17 \%$ pred versus $77 \pm 19 \%$ pred in the combined dataset.

Based on previous studies linking airway bacterial colonisation to impaired MCC [14], we categorised cystic fibrosis subjects according to whether or not they had had a P. aeruginosa-positive $\left(\mathrm{PA}^{+}\right)$sputum or throat culture in the 2 years prior to the imaging study. Of the 37 studies from 24 subjects in the optimal deposition group, 25 were from $16 \mathrm{PA}^{+}$subjects. Table 2 compares $P$. aeruginosa-negative $\left(\mathrm{PA}^{-}\right)$and $\mathrm{PA}^{+}$ subjects. Figure 4 compares MCC in $\mathrm{PA}^{+}$and $\mathrm{PA}^{-}$groups and healthy controls. $\mathrm{PA}^{+}$cystic fibrosis subjects had significantly lower MCC rates than healthy controls $(\mathrm{p}=0.04)$, especially those who were $\mathrm{PA}^{+}$ at their last culture $(\mathrm{p}=0.03) . \mathrm{PA}^{-}$cystic fibrosis subjects had MCC rates similar to healthy controls $(\mathrm{p}=0.62)$. Comparisons between $\mathrm{PA}^{+}$and $\mathrm{PA}^{-}$cystic fibrosis subjects did not demonstrate significant differences, but trended towards decreased MCC, especially with recent $P$. aeruginosa infection. ABS measurements for the same groups are shown in figure 5. ABS was increased in all cystic fibrosis groups when compared to healthy controls and did not vary significantly with $P$. aeruginosa colonisation.

Table 3 considers other correlations between ABS and MCC and patient-related variables, measures of clinical condition and imaging procedure-related measurements. As previously described, central deposition can influence measurements of both MCC and ABS. The use of the optimal deposition group, which excludes subjects with high central levels of deposition, lessens this influence, but does not completely negate it for ABS $(\mathrm{p}=0.10)$.

10 paediatric cystic fibrosis patients were recruited to perform two measurements of ABS and MCC separated by 2 years. Subject data are presented in tables 4 and 5 . One subject was excluded from all analyses based on poor inhalation technique (not listed). Another subject failed to deposit a sufficient radiopharmaceutical lung dose during their second study visit and data from that visit were excluded (subject 7). One subject (subject 3)
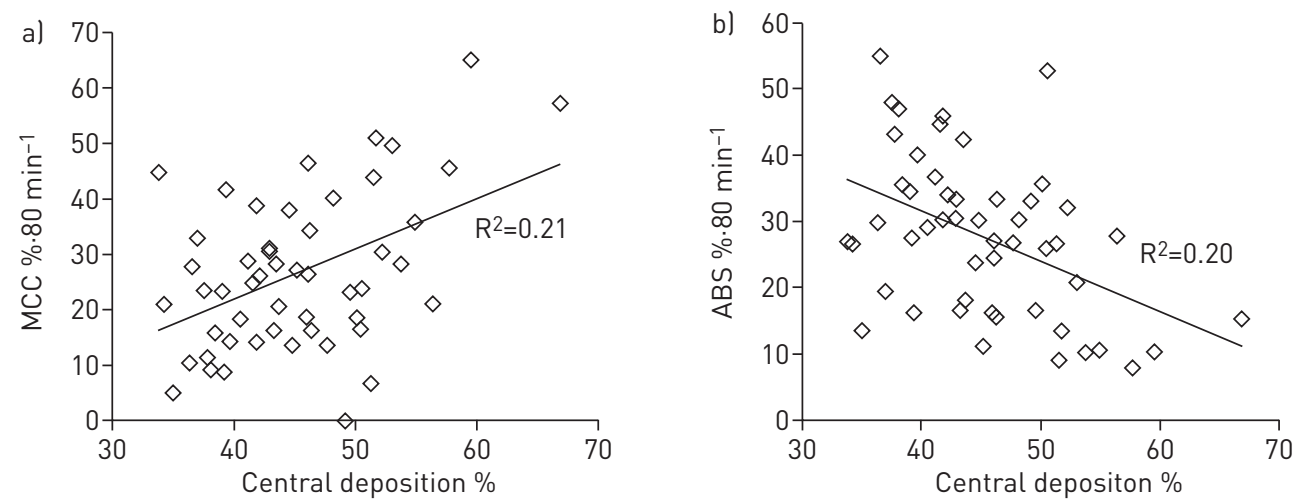

FIGURE 2 Comparison of central lung deposition percentage to measurements of a) mucociliary clearance (MCC) and b) DTPA absorption (ABS). 


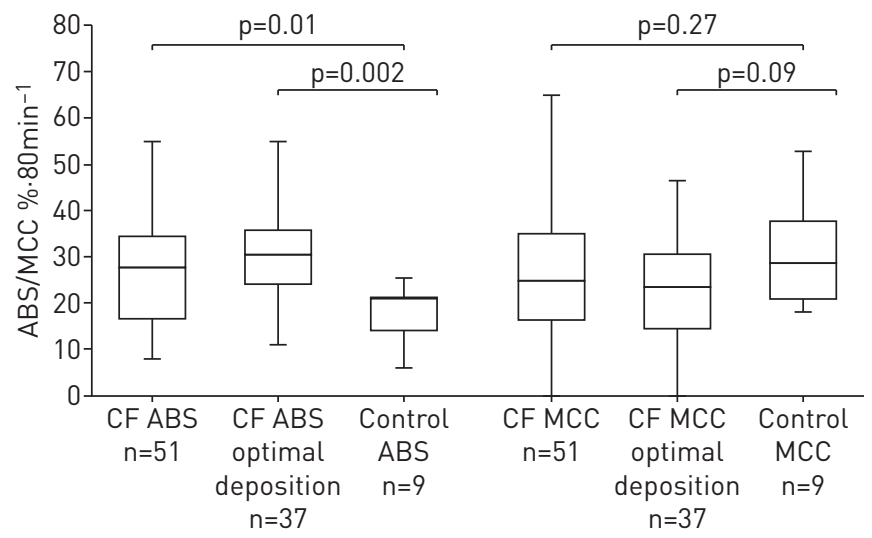

FIGURE 3 Multistudy comparison of measurements of DTPA absorption (ABS) and mucociliary clearance (MCC) in cystic fibrosis (CF) and noncystic fibrosis subjects ( $p$-values calculated using Mann-Whitney test).

was able to perform ABS/MCC tests, but was unable to perform repeatable pulmonary function tests. One subject (subject 4 ) had $>50 \%$ central lung deposition on the second day, and day 2 was excluded from the analysis. MCC/ABS data were therefore available from all nine subjects on visit 1 and seven subjects on visit 2 .

As shown in figure 6, there were no statistical differences between day 1 and day 2 values for MCC $(\mathrm{p}=0.87)$ or $\mathrm{ABS}(\mathrm{p}=0.24)$. Central lung deposition percentage was similar between visits $(\mathrm{p}=0.33)$. On average, $\mathrm{FEV} 1 \%$ pred declined by $0.9 \pm 14 \%$ in this group, while $\mathrm{FEF} 25-75 \% \%$ pred declined by $1.5 \pm 27 \%$ between study visits.

To determine whether MCC or ABS measurements were associated with subsequent clinical course, a number of associations were evaluated. Day 1 MCC measurements were not predictive of 2- or 4-year slopes in FEV1, FVC or FEF25-75\%, nor were they predictive of the number of oral or intravenous antibiotics received during the 2-year period between visits. Day 1 ABS measurements were not predictive of 2-year slopes in FEV1, FVC, FEF25-75\% or antibiotic treatments. They did predict 4-year slopes in FEV1 and $\mathrm{FEF} 25-75 \%$ ( $\mathrm{p}=0.02$ and $\mathrm{p}=0.04$, respectively, by linear regression). Subject age was positively correlated with ABS on study day 1 ( $p=0.05)$, but not on study day 2. Age did not correlate with MCC, central deposition percentage or central/peripheral deposition ratio on either study day. Sex was not associated with differences in MCC or ABS.

Average MCC in studies of paediatric subjects with previous $P$. aeruginosa colonisation was $18.4 \pm 10.6 \% \cdot 80$ $\min ^{-1}$ ( $\mathrm{n}=8$ measurements from five subjects) versus $26.4 \pm 14.6 \% \cdot 80 \mathrm{~min}^{-1}$ in never-infected subjects $(\mathrm{n}=8$ measurements from four subjects; $\mathrm{p}=0.34$ by Mann-Whitney). Average ABS values in these groups were similar ( $\mathrm{p}=0.87$ ). FEV1 and $\mathrm{FEF} 25-75 \%$ were $96 \pm 17 \%$ pred and $85 \pm 32 \%$ pred for $\mathrm{PA}^{+}$subjects versus $89 \pm 11 \%$ pred and $80 \pm 35 \%$ pred for $\mathrm{PA}^{-}$subjects ( $\mathrm{p}=0.34$ and $\mathrm{p}=0.56$ for $\mathrm{FEV} 1$ and $\mathrm{FEF} 25-75 \%$, respectively). Paediatric subject data are included in the results shown in figures 4 and 5.

TABLE 2 Comparison of cystic fibrosis subjects who were Pseudomonas aeruginosa-negative $\left(\mathrm{PA}^{-}\right)$, - positive $\left(\mathrm{PA}^{+}\right)$(during past 2 years), and $\mathrm{PA}^{+}$at last sputum or throat culture

\begin{tabular}{ccccc}
$\mathbf{P A}^{-}$ & $\mathbf{P A}^{+}$ & $\mathbf{P A}^{+}$at last & \multicolumn{2}{c}{$\mathbf{p}^{- \text {-value }}{ }^{\#}$} \\
\cline { 3 - 5 } & & culture & PA $^{+}$versus $\mathbf{P A}^{-}$ & $\mathbf{P A}^{+}$at last culture \\
versus $\mathbf{P A}^{-}$
\end{tabular}

Data are presented as mean \pm SD or $n$, unless otherwise stated. MCC: mucociliary clearance; ABS: DTPA absorption; FEV1: forced expiratory volume in $1 \mathrm{~s}$; FEF25-75\%: forced expiratory flow at $25-75 \%$ of forced vital capacity. " : Mann-Whitney test. 
FIGURE 4 Mucociliary clearance (MCC) in healthy controls and cystic fibrosis (CF) subjects with or without Pseudomonas aeruginosa infection $\left(\mathrm{PA}^{+}\right.$or $\mathrm{PA}^{-}$, respectively) in the past 2 years.

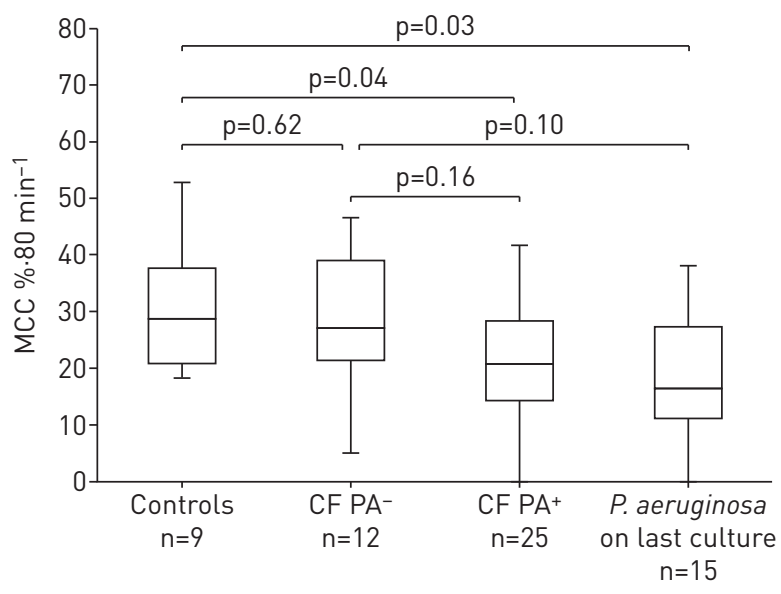

\section{Discussion}

ASL dehydration is a key defect of cystic fibrosis lung disease that is directly linked to CFTR dysfunction. Dehydrated ASL is thought to cause defective MCC, linking CFTR dysfunction to the opportunistic bacterial infections often associated with cystic fibrosis lung disease. The key finding of our study is that defective MCC is present only in cystic fibrosis subjects with a previous history of $P$. aeruginosa infection. Noncolonised subjects demonstrated MCC rates similar to healthy controls. In addition, cystic fibrosis subjects were found to have consistently increased ABS, which may be associated with ASL dehydration. Our studies also indicated potential confounding effects for both MCC and ABS measurements with high central lung aerosol deposition.

Our first goal was to determine the repeatability of the ABS and MCC measurements. Using values for the standard deviation of the intrasubject measurement differences for subjects with similar pulmonary function on both testing days (ABS $\sigma=5.2$ and MCC $\sigma=7.7$ ) we can consider power. For comparison purposes, the inhalation of $7 \%$ hypertonic saline caused a $9.8 \% .80 \mathrm{~min}^{-1}$ decrease in $\mathrm{ABS}$ and an $18.8 \% \cdot 80 \mathrm{~min}^{-1}$ increase in MCC versus the inhalation of isotonic saline [12]. For a crossover study using parametric statistics to estimate power, a group size of 11 is needed to demonstrate a $5 \% \cdot 80 \mathrm{~min}^{-1}$ change in ABS through a paired t-test, assuming that $\sigma=5.2, \Delta=5, \alpha=0.05$ and $\beta=0.2$. Group sizes of seven and 21 are needed to demonstrate $10 \% \cdot 80 \mathrm{~min}^{-1}$ and $5 \% \cdot 80 \mathrm{~min}^{-1}$ changes in MCC, respectively, assuming $\sigma=7.7$, $\Delta=10, \alpha=0.05$ and $\beta=0.2$ [15]. These group sizes are very attractive and feasible for proof-of-concept studies with ASL-modulating drugs.

Aerosol deposition pattern has been previously shown to affect MCC measurements [9], and most current techniques use controlled breathing to promote consistency between studies and deliver aerosols primarily to the large airways [8]. However, obstructive lung disease may cause extremely high rates of central lung aerosol deposition in some subjects [16]. Excessive deposition in the highly ciliated proximal airways will result in very high MCC measurements that make accurate measurement of ABS impossible as the In-DTPA probe clears through MCC too rapidly to absorb. Here, in order to determine the specific patient and disease factors affecting baseline MCC/ABS, we utilised an analysis group with a central deposition

FIGURE 5 DTPA absorption (ABS) in healthy controls and cystic fibrosis subjects (CF) with or without Pseudomonas aeruginosa infection $\left(\mathrm{PA}^{+}\right.$or $\mathrm{PA}^{-}$, respectively) in the past 2 years.

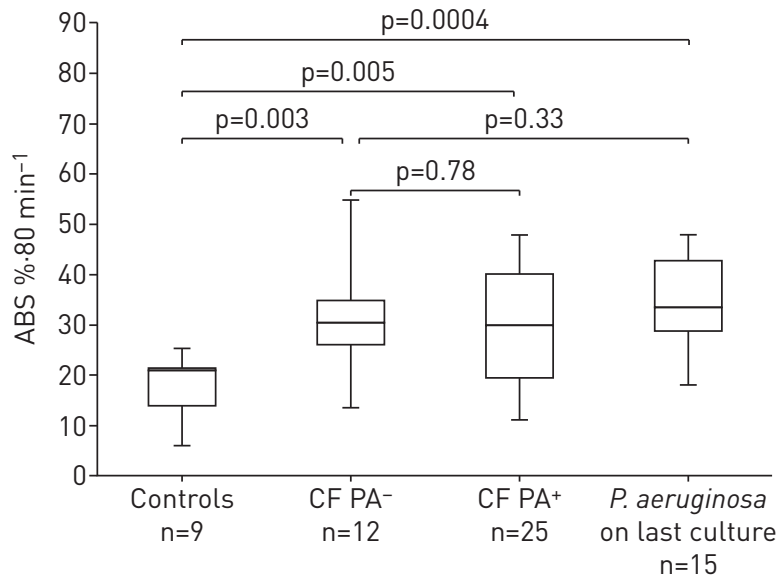


TABLE 3 Sex comparison and linear regression analysis of imaging outcomes

\begin{tabular}{|c|c|c|c|c|}
\hline & Male & Female & Coefficient $(95 \% \mathrm{CI})$ & p-value ${ }^{\#}$ \\
\hline Subjects $\mathrm{n}$ & 22 & 15 & & \\
\hline MCC $\% .80 \mathrm{~min}^{-1}$ & $21.0 \pm 11.0$ & $27.0 \pm 11.4$ & & 0.14 \\
\hline ABS $\% .80 \min ^{-1}$ & $31.4 \pm 11.9$ & $28.4 \pm 9.1$ & & 0.26 \\
\hline \multicolumn{5}{|l|}{ Univariate linear regression } \\
\hline \multicolumn{5}{|l|}{ MCC versus } \\
\hline ABS & & & $-0.18(-0.53-0.18)$ & 0.32 \\
\hline $\mathrm{c} / \mathrm{p}$ ratio & & & $-2.74(-13.86-8.38)$ & 0.62 \\
\hline Central deposition $\%$ & & & $0.20(-0.71-1.11)$ & 0.66 \\
\hline Age & & & $0.22(-0.12-0.56)$ & 0.20 \\
\hline FEV 1 & & & $0.078(-0.159-0.316)$ & 0.51 \\
\hline FEF25-75\% & & & $0.087(-0.051-0.224)$ & 0.21 \\
\hline Starting technetium-99m counts & & & $-0.0013(-0.005-0.002)$ & 0.44 \\
\hline Starting indium-111 counts & & & $-0.000084(-0.02-0.02)$ & 0.99 \\
\hline \multicolumn{5}{|l|}{ ABS versus } \\
\hline$c / p$ ratio & & & $-7.47(-17.1-2.16)$ & 0.12 \\
\hline Central deposition $\%$ & & & $-0.7(-1.54-0.13)$ & 0.10 \\
\hline Age & & & $0.06(-0.28-0.39)$ & 0.73 \\
\hline FEV 1 & & & $0.09(-0.14-0.31)$ & 0.44 \\
\hline FEF25-75\% & & & $0.1(-0.03-0.23)$ & 0.14 \\
\hline Starting technetium- $99 \mathrm{~m}$ counts & & & $0.0015(-0.002-0.005)$ & 0.32 \\
\hline Starting indium-111 counts & & & $0.0041(-0.01-0.02)$ & 0.57 \\
\hline
\end{tabular}

Data are presented as $\mathrm{n}$ or mean $\pm \mathrm{SD}$, unless otherwise stated. Based on 37 measurements from 24 adult and paediatric cystic fibrosis subjects. MCC: mucociliary clearance; ABS: DTPA absorption; c/p: central/peripheral deposition; FEV1: forced expiratory volume in $1 \mathrm{~s}$; FEF25-75\%: forced expiratory flow at $25-75 \%$ of forced vital capacity. "\#: p-values for sex comparison calculated using Mann-Whitney test; other p-values based on linear regression; ${ }^{\text {I" }}$ subjects with high central deposition percentages (>50\%) are not included in this group.

percentage $<50 \%$. This is not intended to be a general limit to be applied to other studies. In particular, crossover studies involving only MCC measurement, where deposition is well matched between study days, may allow for the evaluation of therapeutic effect even with high central deposition percentages.

We generated a combined baseline dataset of MCC/ABS measurements that included measurements from several recent and previous studies [12]. Previously reported trends were confirmed in this larger group. Specifically, baseline ABS is significantly increased in cystic fibrosis subjects versus healthy controls and baseline MCC is not detectably lower in cystic fibrosis. When analysis was restricted to subjects with optimal deposition (central deposition $<50 \%, 37$ out of 51 measurements), MCC differences between cystic fibrosis and control subjects approached significance and the significance of ABS differences increased.

TABLE 4 Characteristics of paediatric subjects participating 2-year longitudinal studies

\begin{tabular}{|c|c|c|c|c|c|c|c|c|c|}
\hline \multirow[t]{2}{*}{ Subject } & \multicolumn{2}{|c|}{ Age years } & \multirow[t]{2}{*}{ Sex } & \multicolumn{2}{|c|}{ FEV $1 \%$ pred } & \multicolumn{2}{|c|}{ FEF $25-75 \% \%$ pred } & \multicolumn{2}{|c|}{ FEV $1 \%$ pred } \\
\hline & Day 1 & Day 2 & & Day 1 & Day 2 & Day 1 & Day 2 & 4-year" & 2-year \\
\hline 1 & 13.4 & 15.6 & M & 88 & 105 & 65 & 86 & -1.1 & 8.4 \\
\hline 2 & 9.1 & 11.2 & $\mathrm{~F}$ & 106 & 126 & 82 & 135 & -2.8 & 6.1 \\
\hline 3 & 9.6 & 11.8 & $F$ & NA & NA & NA & NA & NA & NA \\
\hline 4 & 12 & 14.1 & $F$ & 103 & 94 & 134 & 120 & 2.1 & 0.6 \\
\hline 5 & 11.6 & 13.7 & $M$ & 78 & 68 & 45 & 35 & -3.0 & -3.9 \\
\hline 6 & 10.6 & 12.6 & $M$ & 94 & 89 & 91 & 63 & -4.0 & -2.8 \\
\hline 7 & 9.2 & 11.2 & $\mathrm{~F}$ & 100 & 104 & 111 & 113 & -1.3 & -2.1 \\
\hline 8 & 12.3 & 14.3 & $M$ & 80 & 76 & 56 & 53 & -0.3 & -2.6 \\
\hline 9 & 11.1 & 13.3 & M & 93 & 73 & 80 & 47 & -2.4 & -3.7 \\
\hline Mean士sD & $11.0 \pm 1.5$ & $13.1 \pm 1.5$ & & $93 \pm 10$ & $92 \pm 20$ & $83 \pm 29$ & $82 \pm 38$ & $-1.6 \pm 1.9$ & $0.0 \pm 4.7$ \\
\hline
\end{tabular}

Data are presented as $n$, unless otherwise stated. FEV1: forced expiratory volume in 1 s; FEF25-75\%: forced expiratory flow at $25-75 \%$ of forced vital capacity; M: male; F: female; NA: not available. ${ }^{\#}$ : 4-year slope of FEV $1 \%$ pred; १: 2-year slope of FEV1 \% pred. 
TABLE 5 Imaging outcomes of paediatric subjects participating in 2-year longitudinal studies

\begin{tabular}{|c|c|c|c|c|c|c|}
\hline \multirow[t]{2}{*}{ Subject } & \multicolumn{2}{|c|}{ MCC $\% .80 \mathrm{~min}^{-1}$} & \multicolumn{2}{|c|}{ ABS $\% .80 \mathrm{~min}^{-1}$} & \multicolumn{2}{|c|}{ Central deposition \% } \\
\hline & Day 1 & Day 2 & Day 1 & Day 2 & Day 1 & Day 2 \\
\hline 1 & 24.9 & 16.4 & 44.8 & 33.4 & 41.6 & 46.3 \\
\hline 2 & 33.0 & 13.7 & 19.5 & 30.4 & 37.0 & 44.8 \\
\hline 3 & 10.5 & 20.7 & 30.0 & 18.2 & 36.3 & 43.7 \\
\hline 4 & 27.9 & EX & 55.0 & EX & 36.5 & 51.3 \\
\hline 5 & 13.6 & 5.1 & 26.8 & 13.6 & 47.7 & 5.0 \\
\hline 6 & 38.8 & 46.5 & 30.3 & 27.2 & 41.9 & 46.1 \\
\hline 7 & 40.2 & EX & 30.4 & EX & 48.2 & EX \\
\hline 8 & 0.0 & 28.3 & 33.3 & 42.5 & 49.2 & 43.5 \\
\hline 9 & 15.9 & 23.4 & 35.7 & 34.6 & 38.4 & 39.0 \\
\hline Mean \pm sD & $22.8 \pm 13.7$ & $22.0 \pm 13.1$ & $34.0 \pm 10.4$ & $28.6 \pm 9.9$ & $41.9 \pm 5.3$ & $43.7 \pm 4.9$ \\
\hline
\end{tabular}

Data are presented as $n$, unless otherwise stated. MCC: mucociliary clearance; ABS: DTPA absorption; EX: excluded (see text).

The effect of $P$. aeruginosa colonisation on MCC was previously considered by LAUBE et al. [14]. In that study, MCC with included cough clearance was depressed in children with $P$. aeruginosa colonisation when compared to those without. Controversy has existed about whether cystic fibrosis subjects demonstrate baseline deficits in 60-90 min measurements of MCC, with some studies demonstrating no differences versus healthy controls $[6,12]$ and others demonstrating clear deficits [17, 18]. These differences are probably related to $P$. aeruginosa status, since the latter group of studies enrolled only $\mathrm{PA}^{+}$ subjects. (This was also the case for several other studies utilising MCC to evaluate therapeutics [7, 19].) One of these studies did identify two $\mathrm{PA}^{-}$subjects as having normal clearance, but did not include them in the final analysis [18]. Some studies have noted an MCC defect in cystic fibrosis using techniques designed to assess peripheral lung MCC, such as 24-h measurements [6]. Shorter measurement periods, including those used here, are dominated by large airway clearance. Our results indicate that $\mathrm{PA}^{-}$cystic fibrosis subjects have MCC rates very similar to healthy controls while $\mathrm{PA}^{+}$cystic fibrosis subjects have MCC rates that are significantly decreased versus controls. MCC differences between the $\mathrm{PA}^{+}$and $\mathrm{PA}^{-}$ cystic fibrosis groups were not statistically significant, but trends indicated a likely association between MCC depression and recent $P$. aeruginosa infection.

MCC may be depressed by $P$. aeruginosa through multiple mechanisms. Ciliary beat frequency can be reduced after exposure to $P$. aeruginosa $[20,21]$, potentially mediated by virulence factors such as pyocyanin [22] and rhamnolipids [23]. Previous studies have demonstrated that mucus production, composition and rheology could be affected by P. aeruginosa [24], and pyocyanin in particular is associated with goblet cell hyperplasia and mucus hypersecretion [25]. As a host response to pathogens in healthy airways, CFTR facilitates ASL secretion, increasing the transportability of mucus. The failure of this mechanism in cystic fibrosis, compounded by infection-driven increases in mucus production, could
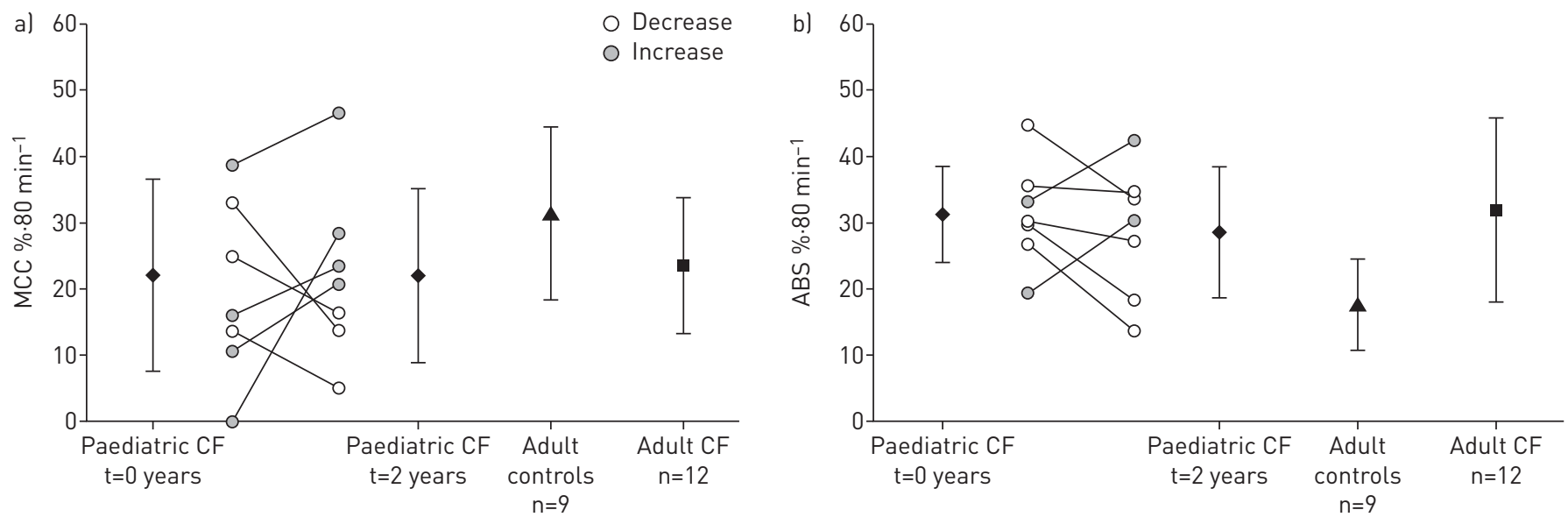

FIGURE 6 Longitudinal measures of a) mucociliary clearance (MCC) and b) DTPA absorption (ABS) in paediatric cystic fibrosis (CF) patients over a 2-year period. Previous data from adult cystic fibrosis and adult control subjects are included for comparison [12]. Data are presented as mean \pm SD. 
defeat otherwise normal rates of MCC in the cystic fibrosis lung. Acute impairments of clearance may extend beyond P. aeruginosa. We can hypothesise that other bacterial or viral infections may depress MCC in cystic fibrosis leading to vulnerability to secondary infections, including $P$. aeruginosa or other cystic fibrosis pathogens. In support of this, previous studies have associated initial P. aeruginosa infection with respiratory syncytial virus and other upper respiratory infections [26, 27]. However, the cause-and-effect relationship between $P$. aeruginosa and depressed clearance cannot be clearly determined from our data. Depressed MCC could be the direct cause of $P$. aeruginosa infection, in line with classic descriptions of cystic fibrosis lung pathophysiology. Loss of this host defence mechanism would certainly make subjects more vulnerable to infection. Another possibility is that airway conditions, such as mucus plugging and associated local hypoxia, could independently predispose subjects to both depressed MCC and $P$. aeruginosa infection.

In longitudinal studies of MCC/ABS in paediatric subjects, there was no indication that ABS or MCC predicted clinical outcomes over a 2-year interval. Infection status and age may have confounded our ability to determine these relationships. The role of $P$. aeruginosa infection has already been described and trends within the paediatric group matched those of the larger analysis group in this regard. Ultimately the small sample size and the use of only a single follow-up measurement probably decreased our ability to detect any subtle effects with these measurements.

Many of the limitations of the current studies are associated with the inherent variability of the aerosol-based techniques applied. We have confirmed the previously described potential for confounding based on changes in aerosol deposition pattern [9]. In addition, our techniques rely on two-dimensional planar imaging of an inherently complex three-dimensional organ that contains large and small airways and alveoli. Our aerosol delivery techniques are intended to target deposition primarily to large airways, but some alveolar contribution to outcomes is likely. Limitations to consider with regard to our analysis of baseline relationships between MCC/ABS and disease state include the use of multiple measurements from some subjects. Our primary analysis group included 37 measurements from 24 subjects obtained through several different protocols that utilised identical techniques. The time between measurements varied from a minimum of $\sim 30$ days to $\geqslant 2$ years. Since each of these studies was associated with a unique set of variables describing patient condition and ABS/MCC, we opted to utilise all measurements. The study was also limited by having only a small group of control subjects for comparison.

In summary, measurements of MCC and ABS demonstrated good repeatability. The primary experimental factor affecting the measurements is the extent of central deposition. Excluding subjects with high central deposition appeared to increase the statistical significance of observed trends. The primary patient factor associated with depressed MCC is $P$. aeruginosa infection. $\mathrm{PA}^{-}$cystic fibrosis subjects had MCC rates similar to healthy controls while $\mathrm{PA}^{+}$cystic fibrosis subjects had depressed MCC, apparently more so with recent infection. Neither ABS nor MCC predicted the clinical course of disease in a small number of paediatric cystic fibrosis subjects though a 2-year follow-up. Multiple longitudinal measurements may provide more comprehensive information on disease evolution and the factors leading to initial $P$. aeruginosa colonisation. We hypothesise that defects in ASL secretion in the cystic fibrosis airway result in clearance that is not depressed at baseline, but rather is vulnerable to depression by factors such as infection that in turn might lead to cycles of secondary infection and disease progression.

\section{References}

1 Collins FS. Cystic fibrosis: molecular biology and therapeutic implications. Science 1992; 256: 774-779.

2 Matsui H, Grubb BR, Tarran R, et al. Evidence for periciliary liquid layer depletion, not abnormal ion composition, in the pathogenesis of cystic fibrosis airways disease. Cell 1998; 95: 1005-1015.

3 Boucher RC. Evidence for airway surface dehydration as the initiating event in CF airway disease. J Intern Med 2007; 261: 5-16.

4 Blouquit S, Regnier A, Dannhoffer L, et al. Ion and fluid transport properties of small airways in cystic fibrosis. Am J Respir Crit Care Med 2006; 174: 299-305.

5 Rowe SM, Heltshe SL, Gonska T, et al. clinical mechanism of the cystic fibrosis transmembrane conductance regulator potentiator ivacaftor in G551D-mediated cystic fibrosis. Am J Respir Crit Care Med 2014; 190: 175-184.

6 Donaldson SH, Bennett WD, Zeman KL, et al. Mucus clearance and lung function in cystic fibrosis with hypertonic saline. N Engl J Med 2006; 354: 241-250.

7 Robinson M, Daviskas E, Eberl S, et al. The effect of inhaled mannitol on bronchial mucus clearance in cystic fibrosis patients: a pilot study. Eur Respir J 1999; 14: 678-685.

8 Bennett WD, Laube BL, Corcoran T, et al. Multisite comparison of mucociliary and cough clearance measures using standardized methods. J Aerosol Med Pulm Drug Deliv 2013; 26: 157-164.

9 Donaldson SH, Corcoran TE, Laube BL, et al. Mucociliary clearance as an outcome measure for cystic fibrosis clinical research. Proc Am Thorac Soc 2007; 4: 399-405.

10 Corcoran TE, Thomas KM, Myerburg MM, et al. Absorptive clearance of DTPA as an aerosol-based biomarker in the cystic fibrosis airway. Eur Respir J 2010; 35: 781-786.

11 Corcoran TE, Thomas KM, Brown S, et al. Liquid hyper-absorption as a cause of increased DTPA clearance in the cystic fibrosis airway. EJNMMI Res 2013; 3: 14. 
12 Locke LW, Myerburg MM, Markovetz MR, et al. Quantitative imaging of airway liquid absorption in cystic fibrosis. Eur Respir J 2014; 44: 675-684.

13 Markovetz MR, Corcoran TE, Locke LW, et al. A physiologically-motivated compartment-based model of the effect of inhaled hypertonic saline on mucociliary clearance and liquid transport in cystic fibrosis. PloS One 2014; 9: e111972.

14 Laube BL, Sharpless G, Benson J, et al. Mucus removal is impaired in children with cystic fibrosis who have been infected by Pseudomonas aeruginosa. J Pediatr 2014; 164: 839-845.

15 Dupont WD, Plummer WD Jr. Power and sample size calculations. A review and computer program. Control Clin Trials 1990; 11: 116-128.

16 Ilowite JS, Gorvoy JD, Smaldone GC. Quantitative deposition of aerosolized gentamicin in cystic fibrosis. Am Rev Respir Dis 1987; 136: 1445-1449.

17 Regnis JA, Robinson M, Bailey DL, et al. Mucociliary clearance in patients with cystic fibrosis and in normal subjects. Am J Respir Crit Care Med 1994; 150: 66-71.

18 Robinson M, Eberl S, Tomlinson C, et al. Regional mucociliary clearance in patients with cystic fibrosis. J Aerosol Med 2000; 13: 73-86.

19 Robinson M, Hemming AL, Regnis JA, et al. Effect of increasing doses of hypertonic saline on mucociliary clearance in patients with cystic fibrosis. Thorax 1997; 52: 900-903.

20 Wilson R, Roberts D, Cole P. Effect of bacterial products on human ciliary function in vitro. Thorax 1985; 40: $125-131$.

21 Mallants R, Jorissen M, Augustijns P. Beneficial effect of antibiotics on ciliary beat frequency of human nasal epithelial cells exposed to bacterial toxins. J Pharm Pharmacol 2008; 60: 437-443.

22 Rada B, Leto TL. Pyocyanin effects on respiratory epithelium: relevance in Pseudomonas aeruginosa airway infections. Trends Microbiol 2013; 21: 73-81.

23 Read RC, Roberts P, Munro N, et al. Effect of Pseudomonas aeruginosa rhamnolipids on mucociliary transport and ciliary beating. J Appl Physiol 1992; 72: 2271-2277.

24 Henke MO, John G, Germann M, et al. MUC5AC and MUC5B mucins increase in cystic fibrosis airway secretions during pulmonary exacerbation. Am J Respir Crit Care Med 2007; 175: 816-821.

25 Hao Y, Kuang Z, Walling BE, et al. Pseudomonas aeruginosa pyocyanin causes airway goblet cell hyperplasia and metaplasia and mucus hypersecretion by inactivating the transcriptional factor FoxA2. Cell Microbiol 2012; 14: 401-415.

26 Petersen NT, Høiby N, Mordhorst CH, et al. Respiratory infections in cystic fibrosis patients caused by virus, chlamydia and mycoplasma - possible synergism with Pseudomonas aeruginosa. Acta Paediatr Scand 1981; 70: 623-628.

27 Collinson J, Nicholson KG, Cancio E, et al. Effects of upper respiratory tract infections in patients with cystic fibrosis. Thorax 1996; 51: 1115-1122. 